\title{
Insulin and glucagon levels of umbilical cord blood in appropriate for gestational age - preterm infants with or without postnatal hypoglycemia
}

\author{
Jae Hyun Park, MD', \\ Jin Gon Bae, MD², \\ Shin Kim, MD, \\ Chun Soo Kim, MD', \\ Sang Lak Lee, MD', \\ Heung Sik Kim, MD, PhD ${ }^{1}$ \\ Departments of ${ }^{1}$ Pediatrics, \\ ${ }^{2}$ Obstetrics and Gynecology, and \\ ${ }^{3}$ Immunology, Keimyung University \\ Dongsan Medical Center, Keimyung \\ University School of Medicine, \\ Daegu, Korea
}

\begin{abstract}
Purpose: To determine whether serum insulin and glucagon levels of umbilical cord blood correlate with subsequent postnatal hypoglycemia in appropriate for gestational age (AGA) - preterm infants at different gestational ages (GAs).

Methods: The serum insulin and glucagon levels of umbilical cord blood were measured using magnetic bead based multiplex immunoassay in 69 AGA premature infants, stratified according to GA: GA 23-30 weeks, early preterm ( $E P, n=31) ; G A 31-34$ weeks, late preterm (LP, $n=38)$. Postnatal hypoglycemia was defined as a capillary glucose level $<40 \mathrm{mg} / \mathrm{dL}$ within the first 60 minutes of life, regardless of GA.

Results: The capillary glucose concentration in EP infants $(65.5 \pm 21.2 \mathrm{mg} / \mathrm{dL})$ was significantly higher than that of LP infants $(55.9 \pm 17.3 \mathrm{mg} / \mathrm{dL})(P=0.043)$. The serum glucagon level in EP infants $(44.3 \pm 28.7 \mathrm{pg} / \mathrm{mL})$ was significantly higher than that in LP infants $(28.1 \pm 13.6 \mathrm{pg} / \mathrm{mL})(P=0.006)$. There was not a significant difference in serum insulin level between EP and LP infants $(372.7 \pm 254.2 \mathrm{pg} / \mathrm{mL}$ vs. $372.4 \pm 209.1$ $\mathrm{pg} / \mathrm{mL}, P=0.996)$. There was a significant difference in the serum glucagon level between infants with and without hypoglycemia $(27.7 \pm 8.9 \mathrm{mg} / \mathrm{dL}$ vs. $36.8 \pm$ $24.6 \mathrm{mg} / \mathrm{dL}, P=0.036)$, but not in the serum insulin level $(451.9 \pm 256.9 \mathrm{pg} / \mathrm{mL}$ vs. $357.4 \pm 222.2 \mathrm{pg} / \mathrm{mL}, P=0.211$ ). Postnatal glucose concentration within the first 60 minutes of life had a significant positive correlation with serum glucagon levels $(r=0.256, P=0.034)$, but not with serum insulin levels $(r=-0.020, P=0.867)$.

Conclusion: Lower glucagon levels of cord blood were seen in premature infants with higher GA, which might contribute to the occurrence of postnatal hypoglycemia.
\end{abstract}

Keywords: Insulin, Glucagon, Fetal blood, Hypoglycemia, Premature infant

\section{Introduction}

Fetus is dependent on the mother for energy metabolism and the synthesis of other metabolic substrates ${ }^{1)}$. At birth, the blood glucose concentration in the umbilical vein is $80 \%-90 \%$ of the prevailing maternal blood glucose concentration ${ }^{2,3)}$, and the supply of glucose abruptly ceases. The glucose concentration falls rapidly, reaching a nadir by 1 hour of age and stabilizes by 3 hours of age even in the absence of exogenous glucose infusion ${ }^{3,4)}$. Umbilical cord clamping leads to decrease in insulin levels and an acute surge in circulating counterregulatory hormones (glucagon, catecholamines, cortisol) levels ${ }^{2,5}$. These hormones stimulate hepatic glycogenolysis and gluconeogenesis to maintain the plasma glucose concentration ${ }^{5,6}$. In term neonates, exogenous glucose infusions such as enteral feeding achieve variable suppression of endogenous glucose production ${ }^{7,8)}$. Therefore, the plasma glucose concentration in full-term healthy neonates is maintained at a steady $40-80 \mathrm{mg} / \mathrm{dL}$ and tolerates fasting.
Received: 18 March, 2016

Revised: 13 May, 2016

Address for correspondence:

Heung Sik Kim, MD, PhD

Department of Pediatrics, Keimyung University Dongsan Medical Center; Keimyung University School of Medicine, 56 Dalseong-ro, Jung-gu, Daegu 41931, Korea

Tel: +82-53 -250-7516

Fax: +82-53-250-7833

E-mail:kimhs@dsmc.or.kr 
However, transient hypoglycemia is often a consequence of changes in the metabolic environment in utero or ex utero, influenced by factors, such as maternal diabetes, maternal drug treatment, intrauterine growth restriction, perinatal asphyxia, hypoxia, hypothermia, and infection ${ }^{9,10)}$. However, insulin, glucagon, and human growth hormone levels did not differ from those of infants who remained normoglycemic ${ }^{11,12}$.

Premature infants are at risk of hypoglycemia due to limited glycogen stores, lower gluconeogenic hormones activities, and decreased hormonal responses ${ }^{13-15)}$. Moderate neonatal hypoglycemia in preterm infants can lead to adverse neurodevelopmental outcomes ${ }^{16,17)}$. Bagnoli et al. ${ }^{18)}$ reported that very low gestational age (GA) is characterized by higher glucagon plasma levels and insulin resistance, both of which may be determinants in the pathogenesis of neonatal hyperglycemia. In the literature, information about the insulin and glucagon levels of cord blood in preterm infants with hypoglycemia is lacking.

Therefore, here we aimed to determine whether serum insulin and glucagon levels of umbilical cord blood correlate with subsequent postnatal hypoglycemia in AGA - preterm infants at different GAs.

\section{Materials and methods}

This prospective study was conducted in the perinatal center of Keimyung University Dongsan Medical Center from October 2014 to March 2015. This study enrolled preterm infants born at 23-34 weeks' gestation. GA was estimated from the mother's last menstrual period. Exclusion criteria were being born to a diabetic mother, being small for GA (SGA), having a congenital anomaly, the presence of perinatal asphyxia and early onset sepsis. SGA was defined as a weight below the 10th percentile for GA according to Korean reference for birth weight (BW) by GA and sex ${ }^{19)}$.

A total of 74 AGA infants, born at Keimyung University Dongsan Medical Center, Daegu, South Korea, were enrolled in this study. In all enrolled infants, initial resuscitation practices followed the most recent guideline, which was updated in 2010 by the International Liaison Committee on Resuscitation and the American Heart Association ${ }^{20)}$. Perinatal asphyxia was defined as a 5-minute Apgar score $\leq 5$. Three infants with perinatal asphyxia were excluded.

There were no infants whose mothers receiving intravenous dextrose during delivery. Infants were screened for capillary glucose levels by the reagent strips method within the first 60 minutes of life, before exogenous glucose infusion such as enteral feeding or intravenous dextrose injection. Postnatal hypoglycemia was defined as a capillary glucose level $<40 \mathrm{mg} /$ $\mathrm{dL}$, regardless of GA. All infants who developed postnatal hypoglycemia were administrated an intravenous glucose infusion (bolus of $2 \mathrm{~mL} / \mathrm{kg} \mathrm{10 \%} \mathrm{dextrose,} \mathrm{and} \mathrm{continuous}$ glucose infusion at $4-7 \mathrm{mg} / \mathrm{kg} / \mathrm{min}$ ). Two infants with persistent hypoglycemia were excluded.

Sixty-nine infants were stratified according to GA: GA 23-30 weeks, early preterm (EP, n=31); GA 31-34 weeks, late preterm (LP, $\mathrm{n}=38$ ).

The following potentially confounding variables were analyzed: GA, BW, ponderal index, delivery mode, body temperature at admission, antenatal steroid, premature rupture of membrane (PROM), respiratory distress syndrome (RDS), 1and 5-minute Apgar scores. All infants with RDS were given an exogenous surfactant replacement via endotracheal intubation prior to the screening for capillary glucose levels.

\section{Analytical procedures}

Umbilical cord blood was collected from the vein of a double-clamped segment of the umbilical cord, into a Vacutainer rapid serum tube (BD Bioscience, San Jose, CA, USA). The serum of umbilical cord blood was immediately separated by cold centrifugation, divided into aliquots, and stored at $-20{ }^{\circ} \mathrm{C}$ until processing. Insulin and glucagon levels of umbilical cord blood were measured by commercially available multiplex immunoassays (Luminex 200, Merck Millipore Corp., Darmstadt, Germany). The multiplex panels used in this study were Human Metabolic Hormone Magnetic Bead Panel (Cat. \#HMHEMAG-34K-03) (Merck Millipore Corp., Darmstadt, Germany). The assays were performed according to the manufacturer's instructions. The capillary glucose concentrations were measured using the reagent strips method from Accu-Chek Performa (Roche Diagnostics, Indianapolis, IN, USA) within the first 60 minutes of life (range, 20-60 minutes).

\section{Statistical analysis}

Results are given as mean \pm standard deviation. Comparisons of demographic and clinical characteristics were performed using the chi-square test or Fisher exact test for categorical variables, and Student $t$-test for continuous variables. The Pearson correlation coefficient was used to analyze the data, and level of significance was set at $a \leq 0.05(P \leq 0.05)$. A $P<0.05$ was considered statistically significant. The IBM SPSS Statistics ver. 20.0 (IBM Co., Armonk, NY, USA) was used for the analyses.

\section{Ethics statement}

This study was approved by the Keimyung University Dongsan Medical Center Institutional Review Board (approval number: 2014-09-007). The need for informed consent was waived by the board because of the prospective study nature.

\section{Results}

Table 1 lists the clinical characteristics between EP and LP infants. The mean GA and BW in the EP group was significantly lower than that of the LP group $(P=0.000)$. However, the ponderal index did not differ between the 2 groups $(P=0.539)$. The mean body temperature at admission of the EP group was significantly higher than that of the LP group $\left(36.1{ }^{\circ} \mathrm{C} \pm 0.3{ }^{\circ} \mathrm{C}\right.$ vs. $\left.35.8^{\circ} \mathrm{C} \pm 0.4^{\circ} \mathrm{C}, P=0.000\right)$. Both 1 - and 5 -minute Apgar score 
in the EP group was significantly lower than those of the LP group $(P<0.05)$. The use of antenatal steroid in the EP group was significantly higher than that in the LP group $(P=0.016)$, but the incidence of PROM was not $(P=0.271)$. The incidence of RDS in the EP group was significantly higher than that in the LP group (100.0\% [31 of 31] vs. 50.0\% [19 of 38], $P=0.000$ ). However, the mean capillary glucose concentration within the first $60 \mathrm{~min}$ of life in LP infants with RDS $(56.4 \pm 20.6 \mathrm{mg} / \mathrm{dL}$, $\mathrm{n}=19)$ was not significantly different from that in LP infants without RDS ( $55.4 \pm 13.7 \mathrm{mg} / \mathrm{dL}, \mathrm{n}=19)(P=0.859)$. The incidence of postnatal hypoglycemia in EP infants $(12.9 \%, 4$ of 31$)$ was lower than in LP infants (27.0\%, 7 of 38), but the difference was not statistically significant $(P=0.533)$.

Postnatal hypoglycemia developed in 11 infants (15.9\%). Table 2 lists the differences between infants with and without postnatal hypoglycemia. The use of antenatal steroid, the incidence of RDS, mean body temperature at admission, 1-min

Table 1. Clinical characteristics of the study group by gestational age

\begin{tabular}{lccc}
\hline Characteristic & $\begin{array}{c}\text { Early preterm } \\
(\mathrm{n}=31)\end{array}$ & $\begin{array}{c}\text { Late preterm }^{\mathrm{b})} \\
(\mathrm{n}=38)\end{array}$ & P-value \\
\hline Gestational age (wk) & $26.7 \pm 2.3$ & $33.0 \pm 1.1$ & 0.000 \\
Birth weight (g) & $1059 \pm 292$ & $1992 \pm 249$ & 0.000 \\
Ponderal index & $2.26 \pm 0.27$ & $2.25 \pm 0.24$ & 0.539 \\
Male sex & $14(45.2)$ & $20(52.6)$ & 0.537 \\
C-sec & $23(74.2)$ & $30(78.9)$ & 0.265 \\
Antenatal steroid & $28(90.3)$ & $25(65.8)$ & 0.016 \\
PROM & $18(58.1)$ & $17(44.7)$ & 0.271 \\
Body temperature ('C) & $36.1 \pm 0.3$ & $35.8 \pm 0.4$ & 0.000 \\
Apgar score & & & \\
1 Minute & $5.5 \pm 1.4$ & $6.8 \pm 1.1$ & 0.000 \\
5 Minutes & $7.5 \pm 1.0$ & $8.3 \pm 0.7$ & 0.000 \\
RDS & $31(100)$ & $19(50.0)$ & 0.000 \\
\hline Values are pres
\end{tabular}

Values are presented as mean \pm standard deviation or number (\%). GA, gestational age; PROM, premature rupture of membrane; RDS, respiratory distress syndrome.

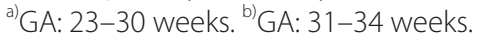

and 5-minute Apgar scores did not differ significantly between the 2 groups $(P>0.05)$.

The serum insulin and glucagon levels of cord blood between EP $(n=31)$ and LP $(n=38)$ infants are shown in Fig. 1. The serum glucagon levels of cord blood in EP infants $(44.3 \pm 28.7 \mathrm{pg} /$ $\mathrm{mL})$ was higher than those in LP infants $(28.1 \pm 13.6 \mathrm{pg} / \mathrm{mL})$, and the difference was statistically significant $(P=0.006)$. There was not a significant difference in the serum insulin levels of cord blood between EP and LP infants $(372.7 \pm 254.2 \mathrm{pg} / \mathrm{mL}$ vs. $372.4 \pm 209.1 \mathrm{pg} / \mathrm{mL}$, respectively, $P=0.996)$. The capillary glucose concentration within the first 60 minutes of life in EP infants $(65.5 \pm 21.2 \mathrm{mg} / \mathrm{dL})$ was significantly higher than those of LP infants $(55.9 \pm 17.3 \mathrm{mg} / \mathrm{dL})$, and the difference was statistically significant $(P=0.043)$.

As shown in Fig. 2, there were significant differences in the serum glucagon levels of cord blood between infants with and without hypoglycemia $(27.7 \pm 8.9 \mathrm{pg} / \mathrm{mL}$ vs. $36.8 \pm 24.6 \mathrm{mg} / \mathrm{dL}$, respectively; $P=0.036$ ), but in the serum insulin levels of cord blood $(451.9 \pm 256.9 \mathrm{pg} / \mathrm{mL}$ vs. $357.4 \pm 222.2 \mathrm{pg} / \mathrm{mL}$, respectively; $P=0.211)$.

An analysis of the linear correlation with capillary glucose concentration within the first 60 minutes of life is shown in Table 3. The capillary glucose concentration within the first 60 minutes of life in AGA-preterm infants was positively correlated

Table 3. Correlation between capillary glucose concentration within the first 60 minutes of life and other parameters in preterm infants

\begin{tabular}{lcc}
\hline Variable & Pearson coefficient & $P$-value \\
\hline Serum glucagon of cord blood & 0.256 & 0.034 \\
Serum insulin of cord blood & -0.020 & 0.867 \\
Gestational age & -0.236 & 0.051 \\
Birth weight & -0.140 & 0.253 \\
Body temperature at admission & 0.204 & 0.093 \\
Apgar score & & \\
1 Minute & -0.151 & 0.215 \\
5 Minutes & -0.219 & 0.071 \\
\hline
\end{tabular}

Table 2. Clinical characteristics of the study group according to postnatal hypoglycemia

\begin{tabular}{|c|c|c|c|}
\hline Characteristic & Postnatal hypoglycemi ${ }^{a}(-)(n=58)$ & Postnatal hypoglycemia ${ }^{b}(+)(n=11)$ & $P$-value \\
\hline Gestational age (wk) & $30.2 \pm 3.6$ & $30.3 \pm 3.5$ & 0.921 \\
\hline Birth weight (g) & $1583 \pm 561$ & $1517 \pm 627$ & 0.727 \\
\hline Ponderal index & $2.25 \pm 0.24$ & $2.29 \pm 0.31$ & 0.609 \\
\hline Male sex & $31(53.4)$ & $3(27.3)$ & 0.111 \\
\hline C-sec & $43(74.1)$ & $10(90.9)$ & 0.227 \\
\hline Antenatal steroid & $43(74.1)$ & $10(90.9)$ & 0.227 \\
\hline PROM & $31(53.4)$ & $4(36.4)$ & 0.299 \\
\hline Body temperature ('C) & $35.9 \pm 0.4$ & $36.0 \pm 0.3$ & 0.537 \\
\hline \multicolumn{4}{|l|}{ Apgar score } \\
\hline 1 Minute & $6.2 \pm 1.4$ & $6.4 \pm 1.6$ & 0.709 \\
\hline 5 Minutes & $8.0 \pm 0.9$ & $7.8 \pm 0.9$ & 0.594 \\
\hline RDS & $41(70.7)$ & $9(81.8)$ & 0.449 \\
\hline
\end{tabular}

Values are presented as mean \pm standard deviation or number (\%).

$\mathrm{PROM}$, premature rupture of membrane; RDS, respiratory distress syndrome.

${ }^{\text {a) }}$ Glucose $\geq 40 \mathrm{mg} / \mathrm{dL}$. ${ }^{\text {b) }} \mathrm{Glucose}<40 \mathrm{mg} / \mathrm{dL}$. 
(A)
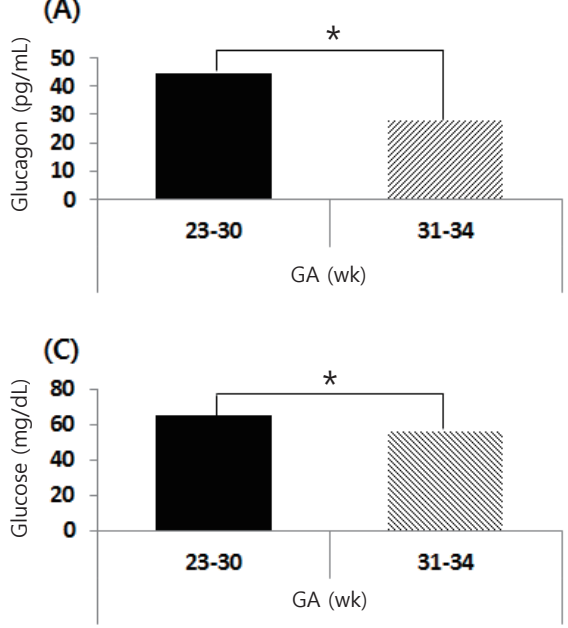

(B)

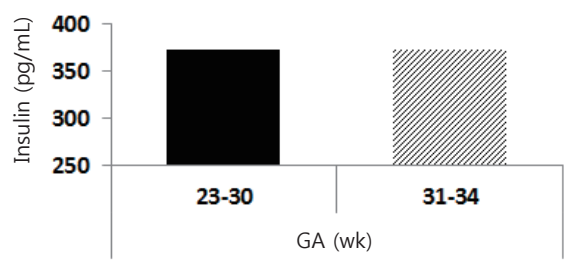

Fig. 1. Serum glucagon (A) and insulin (B) levels in cord blood at birth. (C) The capillary glucose concentrations within the first 60 minutes of life. Glucagon and glucose index were significantly higher in early preterm (GA, 23-30 weeks) than in late preterm (GA, 31-34 weeks) infants. GA, gestational age. ${ }^{*} P<0.05$.
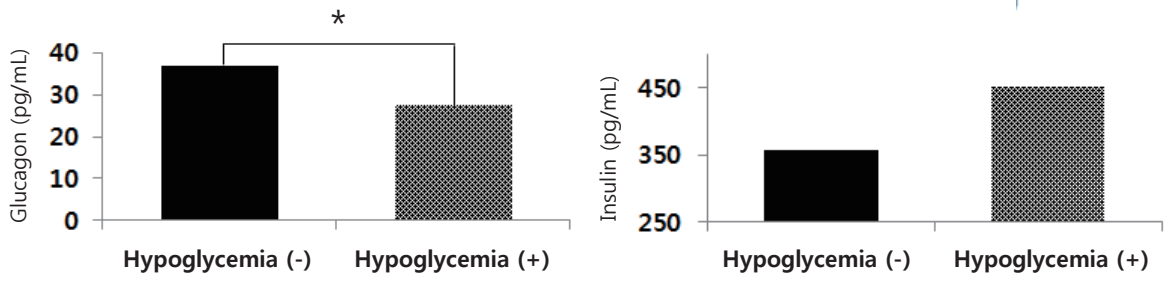

Fig. 2. Serum glucagon and insulin levels of cord blood in preterm infants with and without postnatal hypoglycemia. GA, gestational age. ${ }^{*} P<0.05$

with serum glucagon levels of cord blood $(r=0.256, P=0.034)$, but not with serum insulin levels of cord blood $(r=-0.020$, $P=0.867)$.

\section{Discussion}

The serum glucagon levels of umbilical cord blood in EP infants were significantly higher than those in LP infants. Glucose concentration within the first 60 minutes of life was significantly positively correlated with the serum glucagon levels of cord blood, but not with serum insulin levels. The mean glucose concentration within the first 60 minutes of life in EP infants was higher than that in LP infants. In conclusion, lower glucagon levels in the cord blood were seen in preterm infants with a GA, which might be a determinant of postnatal hypoglycemia.

After birth, transient neonatal hypoglycemia is related to conditions or events occurring during delivery. Perinatal asphyxia is a known risk factor of hyperinsulinism in the neonate owing to the anaerobic metabolism required to maintain blood glucose concentrations ${ }^{21)}$. Infants born to mothers who received intravenous dextrose during delivery ${ }^{22)}$ and were treated with hypoglycemic agents during pregnancy developed postnatal hypoglycemia $^{233}$. SGA infants have a lack of glycogen and fat storage related to the risk for hypoglycemia ${ }^{24)}$. According to previous studies, we excluded these infants from the current study.

Yoon et al. ${ }^{25)}$ reported that the incidence of hypoglycemia (blood glucose concentration $<40 \mathrm{mg} / \mathrm{dL}$ ) during the first 2 hours in preterm infants ( $\mathrm{GA}<34$ weeks) was $12.8 \%$ and that blood glucose levels in the first 1 hour after birth was the highest in the $G A<28$ weeks group. In this present study, the mean glucose concentration within the first 60 minutes of life in the EP infants was higher than that in the LP infants.

Glucagon in the fetus stimulates glucose transfer across the placental barrier. The glucagon receptor is known to maintain maternal-fetal glucose transport as well as intrauterine growth $^{26)}$. The regulation of glucagon release is not obvious ${ }^{27)}$. Bagnoli et al. ${ }^{18)}$ reported that a very low GA was associated with high glucagon levels, which could be due to immaturity of the mechanisms controlling the hormone secretion. In the present study, EP infants with lower GA were associated with high glucagon levels, which could explain why the mean postnatal capillary glucose concentration in EP infants was higher than 
that in LP infants.

Lower GA and lower BW are the risk factor of hypothermia ${ }^{28)}$. However, mean body temperature at admission in EP infants was significantly higher than that in LP infants in this present study. This significant difference in this study was associated with a different transport system from delivery room to neonatal intensive care unit as follows: EP infants transported by radiant warmer versus LP infants transported by a portable incubator without heating. Hypothermia is the risk factor of postnatal hypoglycemia in preterm infants. In the present study, body temperature at admission did not differ significantly between infants with and without postnatal hypoglycemia. Except for enviornmental factors, it could be necessary to investigate the correlation between body temperature and postnatal glucose concentration.

Before preterm delivery, the treatment of antenatal corticosteroids decreases the neonatal mortality and morbidites such as RDS, intaventricular hemorrhage, bronchopulmonary dysplasia, and necrotizing enterocolitis ${ }^{29)}$. Koivisto et al. ${ }^{30)}$ reported that the long exposure time to antenatal glucocorticoid was associated with the increased risk of postnatal hyperglycemia. In contrast to privious studies. On the contrary, Pettit et al. ${ }^{31)}$ reported that antenatal steroid was associated with postnatal hypoglycemia, which was related to maternal hyperglycemia. In the present study, antenatal steroid administration did not differ significantly between infants with and without postnatal hypoglyemia. However, we did not investigate whether antenatal steroid induced maternal hyperglycemia. Further prospective studies are needed to evaluate the effect of antenatal steroid on postnatal glyemic control.

Padbury et al. ${ }^{32)}$ reported that a surge of catecholamine from the fetal sympathoadrenal system was induced by postpartum stress, such as hypoxia. In this study, all EP infants underwent endotracheal intubation for the surfactant replacement of RDS. This could explain why the mean postnatal capillary glucose concentration in EP infants was higher than that in LP infants. However, further evaluations are needed to clarify whether the neonatal resuscitation affects postnatal glucose concentration.

This study was limited by factors such as a small number of infants and the lack of data on serum insulin and glucagon levels with hypoglycemic events.

In conclusion, lower glucagon levels of cord blood were seen in premature infants with higher GA, which might contribute to the occurrence of postnatal hypoglycemia.

\section{Conflict of interest}

No potential conflict of interest relevant to this article was reported.

\section{Acknowledgments}

This work was supported by the National Research Foundation of Korea (NRF) Grant funded by the Korean Government
(MSIP) (No. 2014R1A5A2010008).

\section{References}

1. Kalhan SC, D'Angelo LJ, Savin SM, Adam PA. Glucose production in pregnant women at term gestation. Sources of glucose for human fetus. J Clin Invest 1979;63:388-94.

2. Bloom SR, Johnston DI. Failure of glucagon release in infants of diabetic mothers. Br Med J 1972;4:453-4.

3. Heck LJ, Erenberg A. Serum glucose levels in term neonates during the first 48 hours of life. J Pediatr 1987;110:119-22.

4. Srinivasan G, Pildes RS, Cattamanchi G, Voora S, Lilien LD. Plasma glucose values in normal neonates: a new look. J Pediatr 1986;109:114-7.

5. Sperling MA, DeLamater PV, Phelps D, Fiser RH, Oh W, Fisher DA. Spontaneous and amino acid-stimulated glucagon secretion in the immediate postnatal period. Relation to glucose and insulin. J Clin Invest 1974;53:115966.

6. Cornblath M, Reisner SH. Blood glucose in the neonate and its clinical significance. N Engl J Med 1965;273:378-81.

7. Cowett RM, Oh W, Schwartz R. Persistent glucose production during glucose infusion in the neonate. J Clin Invest 1983;71:467-75.

8. Kalhan SC, Oliven A, King KC, Lucero C. Role of glucose in the regulation of endogenous glucose production in the human newborn. Pediatr Res 1986;20:49-52.

9. Zhou W, Yu J, Wu Y, Zhang H. Hypoglycemia incidence and risk factors assessment in hospitalized neonates. J Matern Fetal Neonatal Med 2015;28:422-5.

10. Jain A, Aggarwal R, Jeeva Sankar M, Agarwal R, Deorari AK, Paul VK. Hypoglycemia in the newborn. Indian J Pediatr 2010;77:1137-42.

11. Hume R, McGeechan A, Burchell A. Failure to detect preterm infants at risk of hypoglycemia before discharge. J Pediatr 1999;134:499-502.

12. Jackson L, Williams FL, Burchell A, Coughtrie MW, Hume R. Plasma catecholamines and the counterregulatory responses to hypoglycemia in infants: a critical role for epinephrine and cortisol. J Clin Endocrinol Metab 2004;89: 6251-6.

13. Hawdon JM, Ward Platt MP, Aynsley-Green A. Patterns of metabolic adaptation for preterm and term infants in the first neonatal week. Arch Dis Child 1992;67(4 Spec No):357-65.

14. Mitanchez D. Glucose regulation in preterm newborn infants. Horm Res 2007;68:265-71.

15. Lyall H, Burchell A, Howie PW, Ogston S, Hume R. Early detection of metabolic abnormalities in preterm infants impaired by disorders of blood glucose concentrations. Clin Chem 1994;40:526-30.

16. Lucas A, Morley R, Cole TJ. Adverse neurodevelopmental outcome of moderate neonatal hypoglycaemia. BMJ 1988;297:1304-8. 
17. Duvanel CB, Fawer CL, Cotting J, Hohlfeld P, Matthieu JM. Long-term effects of neonatal hypoglycemia on brain growth and psychomotor development in small-forgestational-age preterm infants. J Pediatr 1999;134:492-8.

18. Bagnoli F, Vodo F, Vodo S, Conte ML, Tomasini B, Vodo $Z$, et al. Glucagon and insulin cord blood levels in very preterm, late preterm and full-term infants. J Pediatr Endocrinol Metab 2014;27:419-23.

19. Lim JS, Lim SW, Ahn JH, Song BS, Shim KS, Hwang IT. New Korean reference for birth weight by gestational age and sex: data from the Korean Statistical Information Service (2008-2012). Ann Pediatr Endocrinol Metab 2014;19:14653.

20. Kattwinkel J, Perlman JM, Aziz K, Colby C, Fairchild K, Gallagher J, et al. Part 15: neonatal resuscitation: 2010 American Heart Association Guidelines for Cardiopulmonary Resuscitation and Emergency Cardiovascular Care. Circulation 2010;122(18 Suppl 3):S909-19.

21. Collins JE, Leonard JV. Hyperinsulinism in asphyxiated and small-for-dates infants with hypoglycaemia. Lancet 1984;2:311-3.

22. Carmen S. Neonatal hypoglycemia in response to maternal glucose infusion before delivery. J Obstet Gynecol Neonatal Nurs 1986;15:319-23.

23. Christesen HB, Melander A. Prolonged elimination of tolbutamide in a premature newborn with hyperinsulinaemic hypoglycaemia. Eur J Endocrinol 1998;138:698-701.

24. Lubchenco LO, Bard H. Incidence of hypoglycemia in newborn infants classified by birth weight and gestational age. Pediatrics 1971;47:831-8.
25. Yoon JY, Chung HR, Choi CW, Yang SW, Kim BI, Shin $\mathrm{CH}$. Blood glucose levels within 7 days after birth in preterm infants according to gestational age. Ann Pediatr Endocrinol Metab 2015;20:213-9.

26. Vuguin PM, Kedees MH, Cui L, Guz Y, Gelling RW, Nejathaim M, et al. Ablation of the glucagon receptor gene increases fetal lethality and produces alterations in islet development and maturation. Endocrinology 2006;147:3995-4006.

27. Pipeleers DG, Schuit FC, Van Schravendijk CF, Van de Winkel M. Interplay of nutrients and hormones in the regulation of glucagon release. Endocrinology 1985;117:817-23.

28. Riesenfeld T, Hammarlund K, Sedin G. Respiratory water loss in relation to gestational age in infants on their first day after birth. Acta Paediatr 1995;84:1056-9.

29. Roberts D, Dalziel S. Antenatal corticosteroids for accelerating fetal lung maturation for women at risk of preterm birth. Cochrane Database Syst Rev 2006;(3):CD004454.

30. Koivisto M, Peltoniemi OM, Saarela T, Tammela O, Jouppila $\mathrm{P}$, Hallman M. Blood glucose level in preterm infants after antenatal exposure to glucocorticoid. Acta Paediatr 2007;96:664-8.

31. Pettit KE, Tran SH, Lee E, Caughey AB. The association of antenatal corticosteroids with neonatal hypoglycemia and hyperbilirubinemia. J Matern Fetal Neonatal Med 2014;27:683-6.

32. Padbury JF, Agata Y, Polk DH, Wang DL, Lam RW, Callegari CC. Catecholamine and endorphin responses to delivery in term and preterm lambs. Dev Pharmacol Ther 1988;11:4450 . 